\title{
Distribution of Peak-to-Average Power Ratio and Its Applications in OFDM Systems with Unequal Power Allocation
}

\author{
Tao Jiang and Daiming Qu \\ Department of Electronics and Information Engineering \\ Huazhong University of Science and Technology, China \\ Email: Tao.Jiang@ieee.org
}

\author{
Peng Gao \\ Division of Research \\ China Mobile Group Design Institute Co., Ltd, China \\ Email: gaopeng@cmdi.chinamobile.com
}

\begin{abstract}
In this paper, we propose a general expression of the peak-to-average power ratio (PAPR) distribution in orthogonal frequency division multiplexing (OFDM) systems with unequal power allocation strategy, which is based on the extreme value theory for Chi-squared-2 process. The proposed general distribution of the PAPR depends on the number of the subcarriers, distribution of the power allocation and the amount of the total transmission power. To validate the analytical results, we conduct extensive experiments and the numerical results also show that the proposed distribution of the PAPR has a good agreement with that of OFDM systems with unequal power allocation strategy. Finally, we show an example of that the proposed distribution of the PAPR could be directly applied to design the OFDM systems with selective mapping technique to reduce the PAPR.
\end{abstract}

\section{INTRODUCTION}

Recently, orthogonal frequency division multiplexing (OFDM) techniques are playing more and more important role in wireless broadband transmission over multi-path fading channels due to significant improvement of system performance [1]. However, as one of challenges, large peakto-average power ratio (PAPR) will distort the transmitted signals if the transmitter contains nonlinear components such as digital-to-analog converters (DAC), mixers, high power amplifiers (HPA), which results in spectral spreading, intermodulation distortion and changing of the signal constellation.

Recently, various approaches have been proposed to reduce the PAPR for OFDM systems, including clipping [2]-[5], coding schemes [6]-[12], phase optimization [13], [14], nonlinear companding transforms [15]-[23], tone reservation and tone injection [24], [25], constellation shaping [26]-[28], partial transmission sequence (PTS) and selective mapping (SLM) [29]-[46] and other techniques such as pre-scrambles proposed in [47]. Among these schemes, PTS and SLM techniques are two of the most attractive schemes due to their good system performances without the limitation of the subcarriers number. For the SLM method, the input data sequences are multiplied by each of the phase sequences to generate some alternative input symbol sequences. Each of these alternative input data sequences is made the inverse fast Fourier transform (IFFT) operation, and then the one with the lowest PAPR is selected for transmission [39]. Therefore, the ability of the PAPR reduction of the SLM method depends on both the number of phase factors and the design of the phase factors. However, for example, if we only know the type of high power amplifier (HPA) and the desirable probability of clipping is less than $10^{-4}$ when the saturation point of the HPA is $8.0 \mathrm{~dB}$, how many phase factors do we need for the design of OFDM systems with unequal transmission power allocation strategy? Therefore, it is very significant to examine the PAPR characteristic of the transmitted OFDM signals in OFDM systems. More importantly, it is essential to accurately identify the PAPR distribution in OFDM systems. Commonly, complementary cumulative distribution function (CCDF), which denotes the probability that the PAPR of a data block exceeds a given threshold, is often used to express the statistical PAPR distribution of the OFDM signals [24].

It has been widely accepted that the CCDF is one of the key parameters in the design of OFDM systems, such as predicting the impact of nonlinearities [48]. For HPA, we can select the clipping ratio according to both the saturation point of the HPA and the PAPR distribution in adaptive OFDM systems [49]. We also can warrant the minimum of the total degradation of the HPA by selecting its appropriate output back-off (OBO) according to the PAPR's CCDF of the transmitted signals [50]. Moreover, the PAPR distribution can be directly applied to calculate the bit error rate (BER) [51] and to provide limits on achievable information rates [52]. In practice, we usually adjust these design parameters according to simulation results. However, as indicated in [48], if we can use an analytical expression to accurately calculate the PAPR distribution, it can simplify the produce of the OFDM system design.

Recently, several researchers (for example, see [24], [53], [54], [58], [59] and the references therein) have studied the PAPR distribution/bounds of the OFDM signals. However, existing results were obtained just based on the assumption 
that an equal transmission power is allocated to each subcarrier. In other words, these results may not be valid in OFDM systems when the transmission power allocated to each subcarrier is unequal, which considerably can improve the BER performance according to different subchannel conditions. For example, the water-pouring technique has been proposed to maximize the channel capacity [60]. In [61], a general approach to identify PAPR distribution in OFDM systems has been proposed, which is based on extreme value theory, to produce a more accurate analytical expression of the PAPR distribution in OFDM systems, where the transmission power is only allocated to active subcarriers and no power is allocated to inactive subcarriers. Power allocation is an integral part of MAC protocol design in communication systems and it has been well studied [60], [62], [63]. For example, to save the power effectively in adaptive OFDM system, less transmission power is allocated to the subcarriers with lower received signal-to-noise ratios (SNRs) [64]. Therefore, we feel that it is indeed needed to identify a CCDF expression of the PAPR in OFDM systems with unequal power allocation strategy.

In this paper, we are motivated to derive a general CCDF expression of the PAPR in OFDM systems with unequal power allocation strategy, where we employ the extreme value theory for the envelope of a complex Gaussian random Chi-squared-2 process in OFDM systems. The derived CCDF expression of the PAPR is mainly dependent on the number of subcarriers, and the distribution of the power allocated to subcarriers, which can provide more useful insight for the design of OFDM systems.

The rest of this paper is organized as follows. In Section II, we briefly introduce a typical OFDM system with unequal power allocation strategy. In Section III, we analyze and derive out a general CCDF expression of the PAPR using extreme value theory in OFDM systems. In Section IV, extensive simulation results are conducted to validate the tightness of the derived theoretical CCDF expression of the PAPR in OFDM systems. One application of the proposed CCDF expression is shown in Section V for the design of OFDM systems, followed by conclusions in Section VI.

\section{OFDM SYSTEM WITH UNEQUAL TRANSMISSION POWER ALLOCATION STRATEGY}

It is known that the error probability is dominated by the subcarriers with the highest attenuation since the channels fading on different subchannels are different when a fixed strategy is used to allocate equal transmission power to all subcarriers in OFDM systems. Therefore, commonly, OFDM systems employ adaptive strategies to allocate unequal transmission power to each subcarrier, which depends on the condition of each subchannel. Note that, the goal of all adaptive techniques including bit and power loading algorithms is to find the best performance, so that the overall transmission power could be minimized when the rates and the quality-of-services (QoS) requirements are given in OFDM systems. To simplify the

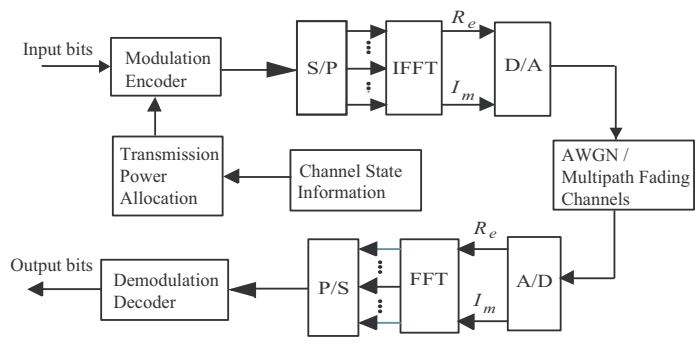

Fig. 1. OFDM system with unequal power allocation strategy.

analysis, we only consider a single user in OFDM systems in this paper.

As shown in Figure 1, the encoder and modulation types are controlled by the transmission power allocation strategy according to the amplitude response of the channel transfer function and other parameters. In other words, the transmission power allocated to each subcarrier is adaptively controlled according to the known channel state information (CSI). In this paper, we assume that each subcarrier has a bandwidth that is much smaller than the coherence bandwidth of the channel, and thus the instantaneous channel gains on all the subcarriers are known to the transmitter.

The complex data at the output of the modulator are transformed into the time domain samples by IFFT operation. Without loss of generality, we consider one symbol interval $t \in\left[0, T_{s}\right]$. Therefore, the equivalent complex baseband signal in OFDM system is expressed as

$$
\begin{aligned}
s_{N}(t) & =x_{N}(t)+j y_{N}(t) \\
& =\frac{1}{\sqrt{N}} \sum_{k=0}^{N-1} A_{k} \exp \left\{j 2 \pi\left(k-\frac{N-1}{2}\right) \frac{t}{T_{s}}\right\},
\end{aligned}
$$

where $x_{N}(t)$ and $y_{N}(t)$ denote the real and imaginary parts of the output signal after the IFFT operation, respectively. $N$ is the number of subcarriers. $A_{k}(k \in \Omega=\{0,1, \ldots, N-1\})$ is the complex symbol modulated by phase shift keying (PSK) or quadrature amplitude modulation (QAM) in a block of $N$ information symbols. Generally speaking, it is often desirable to allocate different amounts of the transmission power $E\left\{\left|A_{k}\right|^{2}\right\}$ to different subcarriers $k$ in OFDM systems, namely, $P_{i} \neq P_{j}$ when $i \neq j(i, j \in \Omega)$.

Commonly, we define that the PAPR of one baseband symbol $s_{N}(t)$ in OFDM systems as

$$
P A P R=10 \log _{10}\left\{\frac{\max _{0 \leq t \leq T_{s}}\left[\left|s_{N}(t)\right|^{2}\right]}{P_{a v}}\right\}(d B),
$$

where $P_{a v}$ is the average power of transmitted signals and it is expressed as follows [24]

$$
P_{a v}=\frac{1}{N} P_{\text {total }}=\frac{1}{N} \sum_{k=0}^{N-1} P_{k},
$$


where $P_{k}=E\left[\left|A_{k}\right|^{2}\right]$ denotes the transmission power allocated to the $k$-th subcarrier and $E\{\cdot\}$ is the expectation.

It is well known that the peak factor of an OFDM output signal $s(t)$ grows linearly with the number of subcarriers in an OFDM system [53]. Moreover, it has been discovered that the PAPR can not give a good measure of the peak power problem when the number of subcarriers $N$ is large, and it is better to use probabilistic analysis, namely, the statistical distribution of the PAPR, i.e., the CCDF of PAPR, in OFDM systems [24], [53].

Recently, some theoretical CCDF approximations of the PAPR have been established in the literature [24], [48], [53], [54], [59]. However, we found that all these existing analytical results have been derived for usual OFDM systems. In other words, they are based on that a constant transmission power is allocated to all subcarriers in OFDM systems, namely, $P_{i}=P_{j}$ for $\forall i, j \in \Omega$. Obviously, this assumption can not be valid in practical OFDM systems since the available quota of transmission energy is distributed to the different bits according to different bit importance to provide optimal unequal error protection in OFDM systems. For example, "water filling" has been widely used in adaptive OFDM systems [62]. Therefore, we feel that it is indeed needed to derive out a theoretical CCDF expression of the PAPR in OFDM systems.

\section{DERIVED Distribution OF PAPR IN OFDM Systems With Unequal POWER Allocation STRATEGY}

\section{A. Convergence to a Gaussian Random Process}

It has been proven that the bandlimited OFDM signal $s_{N}(t)$ with its real part $x_{N}(t)$ and imaginary part $y_{N}(t)$ coverage weakly to Gaussian random processes for any closed and finite interval $T_{s} \subseteq R$ with equal power $P_{i}=P_{j}(\forall i, j \in \Omega)$ allocated to each subcarrier when $N \rightarrow \infty$ [54], [57], namely

$$
\left\{s_{N}(t), t \in T_{s}\right\} \stackrel{D}{\longrightarrow}\left\{s(t), t \in T_{s}\right\},
$$

where $\stackrel{D}{\rightarrow}$ implies the convergence in distribution and $s(t)$ is a zero-mean stationary complex Gaussian random process defined over the symbol duration $T_{s}$ with its autocorrelation function $R_{\left(s_{N}, s_{N}\right)}(\tau)=E\left[s_{N}(t) s_{N}^{*}(t+\tau)\right]$ as follows

$$
\begin{aligned}
& R_{\left(s_{N}, s_{N}\right)}(\tau) \\
& \left.=\frac{1}{N} \sum_{k=0}^{N-1}\left(A_{k} \cdot A_{k}^{*}\right) \exp \left\{j 2 \pi\left(k-\frac{N-1}{2}\right) \frac{\tau}{T_{s}}\right\}\right) \\
& =\frac{1}{N} \sum_{k=0}^{N-1} P_{k} \exp \left(j \omega_{k} \tau\right),
\end{aligned}
$$

where $\omega_{k}=\pi(2 k-N+1) / T_{s}$. It has been proven that $s_{N}(t)$ are uncorrelated with each other. Moreover, when $\tau=$ $0, \pm T_{s}, R_{\left(s_{N}, s_{N}\right)}(\tau)$ always equals $P_{a v}$ for any value of $P_{k}$, which means that $s_{N}(t)$ always converges to an independent stationary Gaussian process whatever the strategy of the power allocation to subcarriers is.
Similarly, when $P_{i} \neq P_{j}(\forall i, j \in \Omega)$ in OFDM systems, it has been established in [24] that the modulated signals can be considered as the sum of $N$ independent and identically distributed (i.i.d) random processes when $\forall k \in \Omega, A_{k}=A_{k}^{R}+$ $j \cdot A_{k}^{I}$ satisfies

$$
\left\{\begin{array}{l}
E\left[A_{k}^{R}\right]=E\left[A_{k}^{I}\right]=0 \\
E\left[A_{k}^{R} A_{k}^{I}\right]=0 \\
E\left[\left|A_{k}^{R}\right|^{2}\right]=E\left[\left|A_{k}^{I}\right|^{2}\right]
\end{array}\right.
$$

Thus, the corresponding autocorrelation functions of $x_{N}(t)$ and $y_{N}(t)$ can be expressed as $R_{\left(x_{N}, x_{N}\right)}(\tau)=$ $E\left[x_{N}(t) x_{N}(t+\tau)\right]$ and $R_{\left(y_{N}, y_{N}\right)}(\tau)=E\left[y_{N}(t) y_{N}(t+\tau)\right]$, respectively, and their cross-correlation function $R_{\left(x_{N}, y_{N}\right)}(\tau)=$ $E\left[x_{N}(t) y_{N}(t+\tau)\right]$ as follows

$$
\begin{aligned}
& R_{\left(x_{N}, x_{N}\right)}(\tau) \\
& =\frac{1}{N} \sum_{k=0}^{N-1}\left[\sigma_{a k}^{2} \cos \left(t_{1}\right) \cos \left(t_{2}\right)+\sigma_{b k}^{2} \sin \left(t_{1}\right) \sin \left(t_{2}\right)\right] \\
& \underline{\underline{\sigma_{a k}^{2}=\sigma_{b k}^{2}}} \frac{1}{2 N} \sum_{k=0}^{N-1} P_{k} \cos \left(\omega_{k} \tau\right), \\
& R_{\left(y_{N}, y_{N}\right)}(\tau) \\
& =\frac{1}{N} \sum_{k=0}^{N-1}\left[\sigma_{a k}^{2} \sin \left(t_{1}\right) \sin \left(t_{2}\right)+\sigma_{b k}^{2} \cos \left(t_{1}\right) \cos \left(t_{2}\right)\right] \\
& \stackrel{\sigma_{a k}^{2}=\sigma_{b k}^{2}}{\underline{1}} \frac{1}{2 N} \sum_{k=0}^{N-1} P_{k} \cos \left(\omega_{k} \tau\right), \\
& R_{\left(x_{N}, y_{N}\right)}(\tau) \\
& \left.=\frac{1}{N} \sum_{k=0}^{N-1}\left\{\sigma_{a k}^{2} \sin \left(t_{2}\right) \cos \left(t_{1}\right)-\sigma_{b k}^{2} \cos \left(t_{2}\right)\right) \sin \left(t_{1}\right)\right\} \\
& \frac{\sigma_{a k}^{2}=\sigma_{b k}^{2}}{\underline{1}} \frac{1}{2 N} \sum_{k=0}^{N-1} P_{k} \sin \left(\omega_{k} \tau\right),
\end{aligned}
$$

where $t_{1}=\omega_{k} t, t_{2}=\omega_{k}(t+\tau), \sigma_{a k}^{2}=E\left\{\left|A_{k}^{R}\right|^{2}\right\}$, and $\sigma_{b k}^{2}=E\left\{\left|A_{k}^{I}\right|^{2}\right\}$ for $\forall k \in \Omega$.

Therefore, it is clear that the following formulations are satisfied

$$
\left\{\begin{array}{l}
R_{\left(s_{N}, s_{N}\right)}(\tau)=2\left[R_{\left(x_{N}, x_{N}\right)}(\tau)+j R_{\left(y_{N}, y_{N}\right)}(\tau)\right], \\
R_{\left(y_{N}, x_{N}\right)}(\tau)=-R_{\left(x_{N}, y_{N}\right)}(\tau)=-R_{\left(y_{N}, x_{N}\right)}(-\tau) .
\end{array}\right.
$$

Therefore, $x_{N}(t)$ and $y_{N}(t)$ are irrelevant for each $t$ and they converge weakly to the stationary processes $x(t)$ and $y(t)$ as $N \rightarrow \infty$, respectively, when $E\left\{\left|A_{k}^{R}\right|^{2}\right\}=E\left\{\left|A_{k}^{I}\right|^{2}\right\}$ and $P_{i} \neq P_{j}$ for $i \neq j(i, j \in \Omega)$. Namely, $x_{N}\left(t_{i}\right)$ and $y_{N}\left(t_{j}\right)$ asymptotically become Gaussian distribution with zero mean and variance $P_{a v} / 2$ for large $N$ according to the central limit theorem, which has been proven in [57].

Similarly, it is easy to prove that the reconstructed signals $\left\{\zeta(t)=\sqrt{2} x_{N}(t) / \sqrt{P_{a v}}, 0 \leq t \leq T_{s}\right\}$ and $\{\eta(t)=$ $\left.\sqrt{2} y_{N}(t) / \sqrt{P_{a v}}, 0 \leq t \leq T_{s}\right\}$ have continuously differentiable 
sample paths and it can be easily proven that $\zeta(t)$ and $\eta(t)$ converge weakly to an independent stationary Gaussian processes with zero mean and unit variance, respectively, when $t>0$ and the number of subcarriers $N$ goes to infinity. Therefore, the identical covariance functions of $\zeta(t)$ and $\eta(t)$ can be written as $r(t)=\operatorname{Cov}(\zeta(t), \zeta(t+\tau))=\operatorname{Cov}(\eta(t), \eta(t+\tau))$, namely

$$
\begin{aligned}
r(t) & =E\left[\frac{2 x(\tau) x(t+\tau)}{P_{a v}}\right]=E\left[\frac{2 y(\tau) y(t+\tau)}{P_{a v}}\right] \\
& =\frac{\sum_{k=0}^{N-1} P_{k} \cos \left(\omega_{k} t\right)}{\sum_{k=0}^{N-1} P_{k}} .
\end{aligned}
$$

B. Proposed CCDF expression of the PAPR in OFDM systems with unequal power allocation

According to the extreme value theory [55]-[57], $r(t)$ satisfies the following expansion

$$
\left\{\begin{array}{l}
r(t)=1-\lambda \frac{t^{2}}{2}+o\left(t^{2}\right), \quad \text { as } t \rightarrow 0, \\
r(t) \log (t) \rightarrow 0, \quad \text { as } t \rightarrow \infty .
\end{array}\right.
$$

Therefore, we can get $\lambda$ with its restriction as follows

$$
\left\{\begin{array}{l}
\lambda=-r^{\prime \prime}(0)=\operatorname{Var}\left(\eta^{\prime}(t)\right)=\operatorname{Var}\left(\zeta^{\prime}(t)\right), \\
\frac{o\left(t^{2}\right)}{t^{2}} \rightarrow 0 \text { as } t \rightarrow 0 .
\end{array}\right.
$$

where $\operatorname{Var}(x)$ denotes the variance of the random variable $x$.

Obviously, $\chi^{2}(t)=\zeta(t)^{2}+\eta(t)^{2}$ is a stationary Chisquared-2 process with continuously differentiable sample paths. Moreover, the conditions stated in (13) are satisfied by $r(t)$ in (11) with

$$
\lambda=\frac{\pi^{2} \sum_{k=0}^{N-1}(2 k+1-N)^{2} P_{k}}{T_{s}^{2} \cdot \sum_{k=0}^{N-1} P_{k}} .
$$

Replacing $t \rightarrow \infty$ with $t^{\prime}=1 / t$, we can easily establish the provable-ness of the second condition stated in (12). According to the extremal theory [55] and Theorem 3.5.1 in [56], then

$$
\operatorname{Prob}\left\{\max _{0 \leq t \leq T_{s}} \chi^{2}(t) \leq \gamma\right\} \stackrel{\gamma, T_{s} \rightarrow \infty}{\longrightarrow} e^{-u} .
$$

If

$$
T_{s} \cdot \mu(\gamma)=T_{s} \cdot e^{\frac{-\gamma}{2}} \sqrt{\frac{\lambda \gamma}{2 \pi}} \stackrel{\gamma, T_{s} \rightarrow \infty}{\longrightarrow} u,
$$

where

$$
u \approx T_{s} \cdot \mu(2 \gamma)=T_{s} \cdot e^{-\gamma} \sqrt{\frac{\lambda \gamma}{\pi}} .
$$

Substituting (14) into (17), we have

$$
u \approx e^{-\gamma} \sqrt{\frac{\pi \gamma \sum_{k=0}^{N-1}(2 k+1-N)^{2} P_{k}}{\sum_{k=0}^{N-1} P_{k}}} .
$$

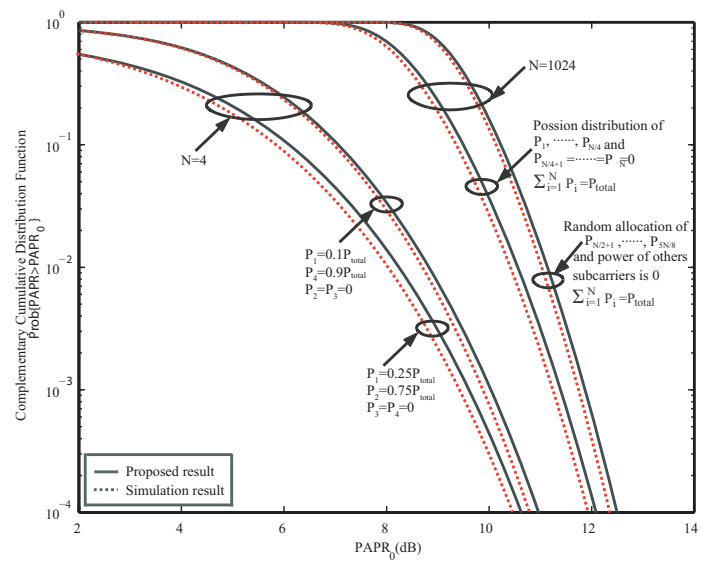

Fig. 2. Comparisons of the PAPR CCDFs with different power allocation strategies in OFDM system with $N=1024$ and $N=4$, respectively.

Hence, as $N \rightarrow \infty$, the cumulative distribution function of the PAPR of the baseband OFDM signal in one symbol interval has the following asymptotic characteristic

$$
\begin{aligned}
& \operatorname{Prob}\{P A P R \leq \gamma\} \\
& =\operatorname{Prob}\left\{\frac{\left|s_{N}^{2}(t)\right|^{2}}{P_{a v}} \leq \gamma\right\} \\
& \cong \operatorname{Prob}\left\{\frac{\max \left\{\left|\zeta^{2}(t)+\eta^{2}(t)\right|^{2}\right\}}{P_{a v}} \leq \gamma\right\} .
\end{aligned}
$$

Therefore, the PAPR CCDF of the OFDM signals can be written as

$$
\begin{aligned}
& \operatorname{Prob}\{P A P R>\gamma\} \cong \\
& 1-\exp \left\{-e^{-\gamma} \sqrt{\frac{\pi \gamma \sum_{k=0}^{N-1}(2 k+1-N)^{2} P_{k}}{\sum_{k=0}^{N-1} P_{k}}}\right\} .
\end{aligned}
$$

for large $N$. Thus, this yields a PAPR distribution of the OFDM symbols with unequal transmission power allocation strategy through a fully analytic derivation.

\section{NUMERICAL RESUlTS}

To get insight into the accuracy of the proposed CCDF expression of the PAPR in OFDM systems with unequal transmission power allocation strategy, extensive computer simulations are conducted, in which $10^{6}$ independent OFDM symbols are randomly generated with an oversampling factor of 4 . The curves labeled by "Simulation result" in all figures are obtained by Monte Carlo searching, and the curves labeled by "Proposed result" in all figures are obtained from the proposed equation (20).

In Figure 2, some results of the PAPR CCDFs are shown when different transmission power allocation strategies are employed in OFDM system with 16-QAM modulation, where 
$N=1024$ and $N=4$, respectively, and only parts of subcarriers are allocated by transmission power. From Figure 2, it is clear that the shapes of the PAPR CCDFs are different when distributions of the transmission power allocation are different. For example, when the $\operatorname{Prob}\left\{P A P R>P A P R_{0}\right\}=10^{-4}$ in the case of $N=1024$, the $P A P R_{0}$ are $12.01 \mathrm{~dB}$ and $12.49 \mathrm{~dB}$, respectively, for the corresponding cases: 1) The distribution of transmission power allocated to $P_{1}, \cdots, P_{N / 4}$ is Possion distribution and the energy allocated to the left subcarriers is 0 ;2) Randomly allocate transmission power to $P_{N / 2+1}, \cdots, P_{5 N / 8}$ and the transmission power of the left subcarriers is 0 . Note that, $\sum_{i=1}^{N}=P_{\text {total }}$ in both cases. Therefore, we can make a conclusion that the PAPR CCDFs are different when the distributions of the powers allocated to subcarriers are different in OFDM systems with unequal power allocation strategies. Obviously, the curve of the proposed approximation (20) is tight to that of simulation result. Moreover, it is distinct that the proposed (20) also agrees well with the simulation result in the case of $N=4$ although the derived (20) is based on large $N$ from Figure 2.

As the frequency domain fading deteriorates the SNR of certain subcarriers, but improves other subcarriers above the average SNR value, the potential loss of throughput due to the exclusion of faded subcarriers can be mitigated by employing higher order modulation modes on the subcarriers exhibiting high SNR values. Therefore, in OFDM system, the goal of adaptive power allocation strategy is to choose the appropriate mode including type of modulation and the number of bits for transmission in each subcarrier when the local SNR is given to achieve a optimal tradeoff between throughput and overall BER. For example, the criterion of modulation decision for each subcarrier has been given in [65] for optimal performance as follows

$$
E_{i}^{m}=\frac{\sigma_{n}^{2} \Gamma_{i}}{\left|H_{i}\right|^{2}}\left(2^{m}-1\right),
$$

where $m \in \mathbf{B}$ is the number of bits assigned to subcarrier by adaptive modulation algorithm, and $\mathbf{B}=\{0,2,4\}$ corresponds to no modulation, QPSK and 16-QAM, respectively. When $m=0$, it means that no bit (no modulation) is needed to be assigned to the subcarrier, i.e., no transmission power is allocated to the subcarrier. When $m=2$, it means that QPSK modulation has been assigned to the subcarrier and $m=4$ means that 16-QAM modulation has been used by the adaptive modulation algorithm. $E_{i}^{m}$ is the needed threshold of the energy for the modulation of the $i$-th subcarrier. $\sigma_{n}^{2}$ is the variance of the Gaussian noise and $H_{i}$ is the channel gain coefficients for the $i$-th subcarrier. $\Gamma_{i}$ is the SNR gap. For simplicity, we set $\Gamma_{i}=1$ for all subcarriers that gives Shannon's famous formula for the channel capacity in this paper.

Figure 3 depicts some results about the CSI and its corresponding transmission power allocated to each subcarrier in adaptive OFDM system with $N=64$, in which the bandwidth is $5.0 \times 10^{6} \mathrm{~Hz}$, the total transmit power is $P=32 \mathrm{mw}$, and the noise power density is $1.0 \times 10^{-8}$. Figure 4 shows the
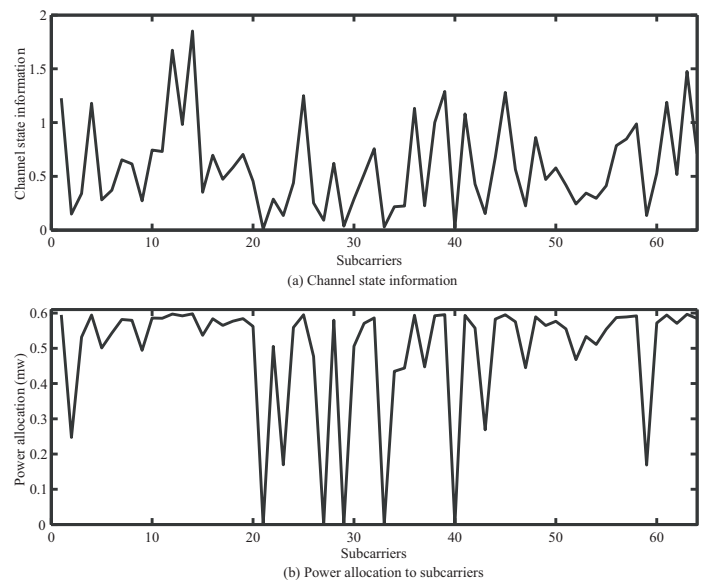

Fig. 3. Unequal transmission power allocated to subcarriers based on waterfilling rule for OFDM system with $N=64$.

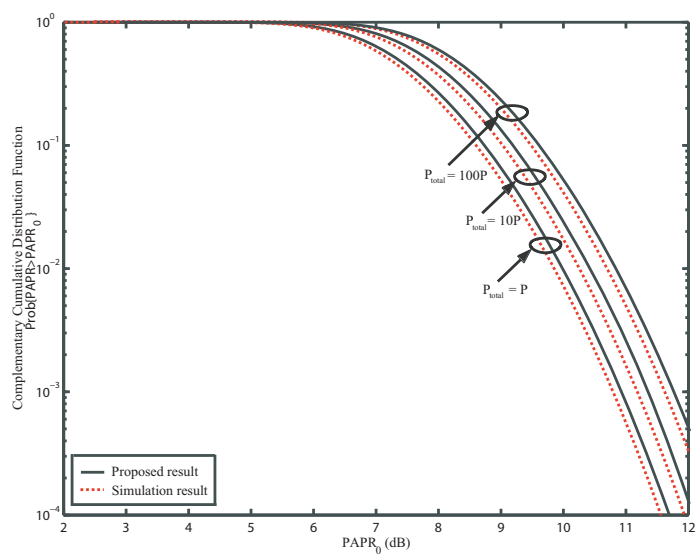

Fig. 4. Comparisons of the PAPR CCDF for OFDM system with different transmission power allocation strategies shown in Fig. 3(b).

results of the PAPR CCDF with unequal transmission power allocation strategies given in Figure 3(b), where 16-QAM is used to modulate the $k$ th subcarrier when its power allocation is larger than or equal to $E_{i}^{4}$, and the QPSK is selected to modulate the $k$ th subcarrier when its transmission power allocation is less than $E_{i}^{4}$ but large than or equal to $E_{i}^{2}$. If there is no transmission power allocated to subcarrier, there is no modulation selected to subcarrier. Moreover, we have conducted some simulations with the amount of total transmission power of $10 P$ and $100 P$, respectively, but the proportion of the transmission power allocated to each subcarrier is the same as that of Figure 3(b) and the corresponding results also have been shown in Figure 4. From Figure 4, we find that: (1) It is evident that the proposed approximation of (20) agrees well with the simulation results; (2) When the amount of the total transmission power allocation is different for a given channel state information, the distribution of the PAPR CCDF 


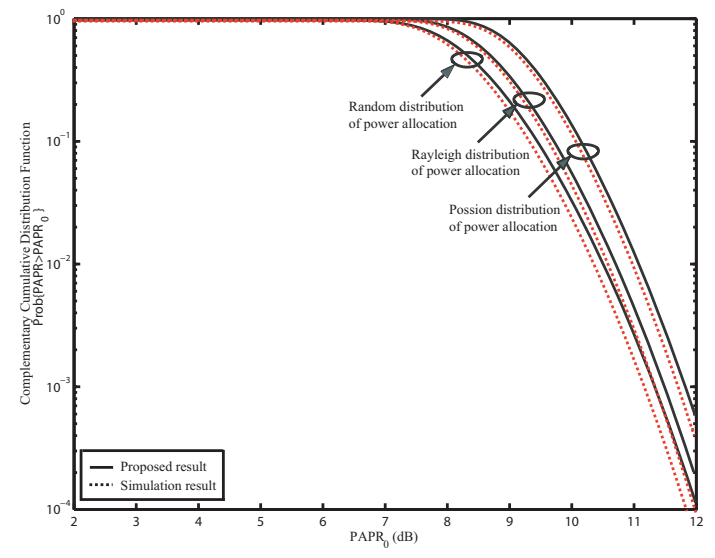

Fig. 5. Comparisons of different PAPR CCDF theoretical and simulated results in OFDM system with unequal power allocation strategies but the same total transmission power $(N=256)$.

is still different although the percentage of the transmission power allocated to each subcarrier is constant. Therefore, we can provide the desirable PAPR CCDF by varying the total transmission power in OFDM systems with unequal power allocation strategies.

Moreover, when the total transmission power is the same but the percentage of the transmission power allocated to each subcarrier is different in OFDM systems, some results are shown in Figure 5, where 16-QAM modulation and $N=$ 256 are deployed. Obviously, the PAPR of the transmitted symbols in OFDM system with the Rayleigh transmission power allocation strategy is much better that of the Possion transmission power allocation strategy, but it is less than that of the Random transmission power allocation strategy.

\section{APPLICATIONS OF THE PROPOSED PAPR CCDF}

It is well known that all the transmitted OFDM signals are input a high power amplifier (HPA), which is peak-power limited, before being transmitted out in a practical OFDM system. In other words, the $s_{N}(t)$ will be clipped if its power is larger than the saturation point of the HPA.

As one of promising techniques, the SLM method takes advantage of the fact that the PAPR of an OFDM signal is only sensitive to phase shifts in the frequency-domain data since its PAPR reduction is achieved by multiplying independent phase sequences with the original data and determining the PAPR of each phase sequence combination. The PAPR CCDF of the OFDM signals with the SLM scheme is simple since $M$ time-domain signals are independent, where $M$ is the number of independent phase sequences. With the independence assumption, the PAPR CCDF of OFDM signals using the SLM technique with unequal transmission power allocated to each

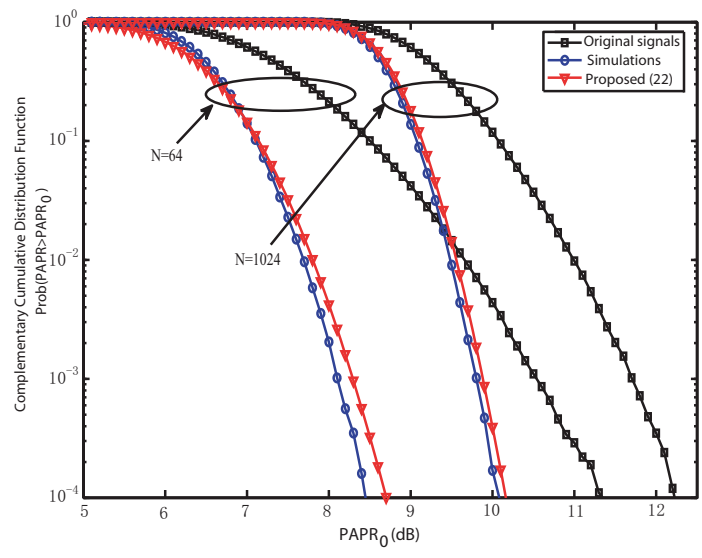

Fig. 6. Comparisons of different PAPR CCDF theoretical and simulated results in OFDM system with unequal power allocation strategy and SLM technique $(\mathrm{M}=4)$

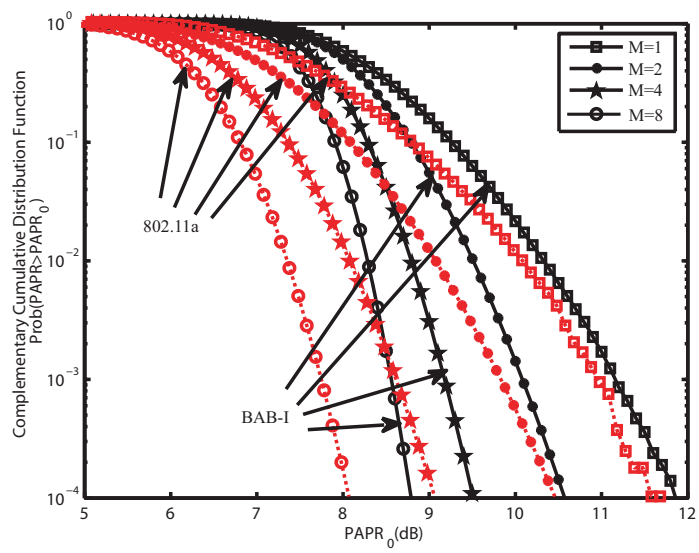

Fig. 7. Results of the proposed PAPR CCDF for 802.11a and DAB-I standards, respectively, using SLM technique ( $M=1,2,4,8$, respectively).

subcarrier can be approximated as

$$
\begin{aligned}
& \operatorname{Prob}\{P A P R>\gamma\} \cong \\
& \left\{1-\exp \left[-e^{-\gamma} \sqrt{\left.\frac{\pi \gamma \sum_{k=0}^{N-1}(2 k+1-N)^{2} P_{k}}{\sum_{k=0}^{N-1} P_{k}}\right]}\right\}^{M} .\right.
\end{aligned}
$$

Figure 6 compares different PAPR CCDF theoretical and simulated results in OFDM system with SLM technique and random allocation of transmission power, where $10^{5}$ independent OFDM symbols are randomly generated with an oversampling factor equaling 4, QPSK modulation, $N=64$ and $N=1024$, respectively. As shown in Figure 6, it is clear that SLM technique can achieve substantial PAPR reduction. Moreover, it is remarkable that the theoretical approximation of (22) has a good match with the simulation results, especially 
when $N$ is large $(N>64)$, although the assumption is not strictly valid because all $M$ of the generated signals have the original frequency-domain data in common. Therefore, we could design some parameters such as $M$ and transmission power allocation strategy when the SLM technique is employed in OFDM systems, which is based on the phenomenon of Figure 6. For example, the proposed PAPR CCDF could be applied to 802.11a WLAN and DAB-I standards as shown in Figure 7. For 802.11a WLAN standard, out of all 64 narrowband subcarriers, only 52 subcarriers are used for data and pilots and the rest 12 subcarriers are null, and thus only these 52 subcarriers are allocated to transmission power randomly and no transmission power is allocated to the left subcarriers. Therefore, for 802.11a WLAN standard, we directly design $M=8$ from Figure 7 to ensure that the probability-of-clipping level of the HPA is less than $10^{-4}$ when the saturation point of the HPA is about $8.0 \mathrm{~dB}$. Similarly, it cannot ensure that the same system performance could be achieved when the same $M$ is directly used, if the same HPA is employed in DAB-I system, which is obvious to find from Figure 7.

\section{CONCLUSIONS}

In this paper, we derived out a general CCDF expression of the PAPR in OFDM systems with unequal transmission power allocation strategy, which is based on the extreme value theory. Extensive computer simulations were conducted to illustrate that the derived expression of the PAPR distribution has a satisfactory accuracy in general and has a good match with the simulation results regardless of the number of subcarriers. The proposed CCDF expression of the PAPR in OFDM systems depends on the number of subcarriers, the distribution of the power allocation to each subcarrier and the total of transmission power. The proposed CCDF expression of PAPR is helpful to provide useful insight for the design of OFDM systems and some examples of application deploying the derived CCDF distribution of the PAPR was illustrated to design the SLM technique to achieve a desirable level of the system performance.

\section{ACKNOWLEDGMENT}

The authors are grateful to Dr. I. J. Wassell from University of Cambridge, for his valuable comments and suggestions, which helped improve the presentation of the paper. This work was supported by the National Science Foundation of China with Grant 60872008, Program for New Century Excellent Talents in University of China under Grant NCET-08-0217, and the Research Fund for the Doctoral Program of Higher Education of the Ministry of Education of China under Grant 200804871142.

\section{REFERENCES}

[1] A. Czylwik. Adaptive OFDM for wideband radio channels; Proc. IEEE Global Telecommunications Conf. (GLOBECOM'96), London, U.K., pp. 713-718, Nov. 1996.
[2] R. O'Neill and L. B. Lopes. Envelope variations and Spectral Splatter in Clipped Multicarrier Signals; Proc. IEEE PIMRC'95, Toronto, canada, pp. 71-75, Sept. 1995.

[3] H. Ochiai and H. Imai. Performance of the deliberate clipping with adaptive symbol selection for strictly band-limited OFDM systems; IEEE Journal on Selected Areas in Communications, vol. 18, no. 11, pp. 2270-2277, Nov. 2000.

[4] S. M. Ju, S. H. Leung. Clipping on COFDM with phase on demand; IEEE Communications Letters, vol. 7, no. 2, pp. 49-51, Feb. 2003.

[5] G. L. Ren, H. Zhang and Y. L. Chang. A complementary clipping transform technique for the reduction of peak-to-average power ratio of OFDM system; IEEE Transactions on Consumer Electronics, vol. 49, no. 4, pp. 922-926, Nov. 2003.

[6] A. E. Jones, T. A. Wilkinson and S. K. Barton. Block Coding Scheme for Reduction of Peak-to-Average Envelope Power Ratio of Multicarrier Transmission Systems; IEE Electronics Letters, vol. 30, no. 8, pp. 20982099, Dec. 1994.

[7] P. Y. Fan; X. G. Xia. Block coded modulation for the reduction of the peak to average power ratio in OFDM systems; IEEE Transactions on Consumer Electronics, vol. 45, no. 4, pp. 1025-1029, Nov. 1999.

[8] D. Wulich, L. Goldfeld. Reduction of peak factor in orthogonal multicarrier modulation by amplitude limiting and coding; IEEE Transactions on Communications, vol. 47, no. 1, pp. 18-21, Jan. 1999.

[9] T. Ginige, N. Rajatheva and K. M. Ahmed. Dynamic spreading code selection method for PAPR reduction in OFDM-CDMA systems with 4-QAM modulation; IEEE Communications Letters, vol. 5, no. 10, pp. 408-410, Oct. 2001.

[10] K. Yang and S. Chang. Peak-to-average power control in OFDM using standard arrays of linear block codes; IEEE Communications Letters, vol. 7, no. 4, pp. 174-176, Apr. 2003.

[11] T. Jiang and G. X. Zhu. Complement block coding for reduction in peak-to-average power ratio of ofdm signals; IEEE Communications Magazine, Vol. 43, No. 9, pp. S17-S22, Sept. 2005.

[12] S. B. Slimane. Reducing the Peak-to-Average Power Ratio of OFDM Signals Through Precoding; IEEE Transactions on Vehicular Technology, vol. 56, no. 2, pp. 686-695, Mar. 2007.

[13] V. Tarokh, H. Jafarkhani. On the computation and reduction of the peakto-average power ratio in multicarrier communications; IEEE Transactions on Communications, vol. 48, no. 1, pp. 37-44, Jan. 2000.

[14] H. Nikookar and K. S. Lidsheim. Random phase updating algorithm for OFDM transmission with low PAPR; IEEE Transactions on Broadcasting, vol. 48, no. 2, pp. 123-128, Jun. 2002.

[15] X. B. Wang, T. T. Tjhung and C. S. Ng. Reduction of peak-to-average power ratio of OFDM system using A companding technique; IEEE Transactions on Broadcasting, vol. 45, no. 3, pp. 303-307, Sept. 1999.

[16] T. Jiang and G. X. Zhu. Nonlinear Companding Transform for Reducing Peak-to-Average Power Ratio of OFDM Signals; IEEE Transactions on Broadcasting, vol. 50, no. 3, pp. 342-346, Sept. 2004.

[17] X. Huang, J. H. Lu, J. L. Zheng, K. B. Letaief and J. Gu. Companding transform for reduction in peak-to-average power ratio of OFDM signals; IEEE Transactions on Wireless Communications, vol. 3, no. 6, pp. 20302039, Nov. 2004.

[18] T. Jiang, Y. Yang and Y. Song. Exponential companding transform for papr reduction in ofdm systems; IEEE Transactions on Broadcasting, vol. 51, no. 2, pp. 244-248, Jun. 2005.

[19] T. Jiang, W. Yao, P. Guo, Y. Song and D. Qu. Two novel nonlinear companding schemes with iterative receiver to reduce papr in multicarrier modulation systems; IEEE Transactions on Broadcasting, vol. 52, no. 2, pp. 268-273, Mar. 2006.

[20] T. G. Pratt, N. Jones, L. Smee and M. Torrey. OFDM link performance with companding for PAPR reduction in the presence of non-linear 
amplification; IEEE Transactions on Broadcasting, vol. 52, no. 2, pp. 261-267, Jun. 2006.

[21] T. Jiang, W. D. Xiang, P. C. Richardson, J. H. Guo and G. X. Zhu. PAPR Reduction of OFDM Signals Using Partial Transmit Sequences with Low Computational Complexity; IEEE Transactions on Broadcasting, vol. 53, no. 3, pp. 719-724, Sept. 2007.

[22] T. Jiang and Y. Wu. An Overview: Peak-to-Average Power Ratio Reduction Techniques for OFDM Signals, IEEE Transactions on Broadcasting, vol. 54, no. 2, pp. 257-268, June. 2008.

[23] T. Jiang, W. Xiang, C. Richardson, D. Qu, and G. Zhu. On the Nonlinear Companding Transform for Reduction in PAPR of MCM Signals, IEEE Transactions on Wireless Communications, vol. 6, no 6, pp. 2017-2021, Jun. 2007.

[24] J. Tellado. Peak to average power ratio reduction for multicarrier modulatio; PhD thesis, University of Stanford, 1999.

[25] S. S. Yoo, S. Yoon, S. Y. Kim and I. Song. A novel PAPR reduction scheme for OFDM systems: selective mapping of partial tones (SMOPT); IEEE Transactions on Consumer Electronics, vol. 52, no. 1, pp. 40-43, Feb. 2006.

[26] Y. Kou; W. S. Lu and A. Antoniou. New peak-to-average power-ratio reduction algorithms for multicarrier communications; IEEE Transactions on Circuits and Systems I: Fundamental Theory and Applications, vol. 51, no. 9, pp. 1790-1800, Sept. 2004.

[27] A. Mobasher, A. K. Khandani. Integer-based constellation-shaping method for PAPR reduction in OFDM systems; IEEE Transactions on Communications, vol. 54, no. 1, pp. 119-127, Jan. 2006.

[28] Y. J. Kou, W. S. Lu, A. Antoniou. A New Peak-to-Average Power-Ratio Reduction Algorithm for OFDM Systems via Constellation Extension; IEEE Transactions on Wireless Communications, vol. 6, no. 5, pp. 18231832, May. 2007

[29] S. H. Muller and J. B. Huber. OFDM with Reduced PeakCtoCAverage Power Ratio by Optimum Combination of Partial Transmit Sequences; IEE Electronics Letters, vol. 33, no. 5, pp. 36-69, Feb. 1997.

[30] S. H. Han, J. H. Lee. PAPR reduction of OFDM signals using a reduced complexity PTS technique; IEEE Signal Processing Letters, vol. 11, no. 11, pp. 887-890, Nov. 2004.

[31] A. Alavi, C. Tellambura, I. Fair. PAPR reduction of OFDM signals using partial transmit sequence: an optimal approach using sphere decoding; IEEE Transactions Communications Letters, vol. 9, no. 11, pp. 982-984, Nov. 2005.

[32] N. T. Hieu, S. W. Kiom, H. G. Ryu. PAPR reduction of the low complexity phase weighting method in OFDM communication system; IEEE Transactions on Consumer Electronics, vol. 51, no. 3, pp. 776-782, Aug. 2005.

[33] L. Yang, R. S. Chen, Y. M. Siu and K. K. Soo. PAPR reduction of an OFDM signal by use of PTS with low computational complexity; IEEE Transactions on Broadcasting, vol. 52, no. 1, pp. 83-86, Mar. 2006.

[34] H. Chen and H. Liang. PAPR Reduction of OFDM Signals Using Partial Transmit Sequences and Reed-Muller Codes; IEEE Communications Letters, vol. 11, no. 6, pp. 528-530, Jun. 2007.

[35] D. H. Park and H. K. Song. A New PAPR Reduction Technique of OFDM System with Nonlinear High Power Amplifier; IEEE Transactions on Consumer Electronics, vol. 53, no. 2, pp. 327-332, May. 2007.

[36] Y. Xiao, X. Lei, Q. Wen and S. Li. A Class of Low Complexity PTS Techniques for PAPR Reduction in OFDM Systems; IEEE Signal Processing Letters, vol. 14, no. 10, pp. 680-683, Oct. 2007.

[37] R. J. Baxley and G. T. Zhou. Comparing Selected Mapping and Partial Transmit Sequence for PAR Reduction; IEEE Transactions on Broadcasting, vol. 53, no. 4, pp. 797-803, Dec. 2007.

[38] S. J. Heo, H. S. Noh, J. S. No and D. J. Shin. A Modified SLM Scheme With Low Complexity for PAPR Reduction of OFDM Systems; IEEE Transactions on Broadcasting, vol. 53, no. 4, pp. 804-808, Dec. 2007.
[39] R. W. Bauml, R. F. H. Fisher and J. B. Huber. Reducing the Peak-toAverage Power Ratio of Multicarrier Modulation by Selected Mapping; IEE Electronics Letters, vol. 32, no. 22, pp. 2056-2057, Oct. 1996.

[40] D. W. Lim, J. S. No, C. W. Lim, H. Chung. A new SLM OFDM scheme with low complexity for PAPR reduction; IEEE Signal Processing Letters, vol. 12, no. 2, pp. 93-96, Feb. 2005.

[41] C. L. Wang and Q. Y. Yuan. Low-complexity selected mapping schemes for peak-to-average power ratio reduction in OFDM systems; IEEE Transactions Signal Processing, vol. 53, no. 12, pp. 4652-4660, Dec. 2005.

[42] G. S. Yue and X. D. Wang. A hybrid PAPR reduction scheme for coded OFDM; IEEE Transactions on Wireless Communications, vol. 5, no. 10, pp. 2712-2722, Oct. 2006.

[43] H. G. Ryu, T. P. Hoa, K. M. Lee, S. W. Kim and J. S. Park. Improvement of power efficiency of HPA by the PAPR reduction and predistortion; IEEE Transactions on Consumer Electronics, vol. 50, no. 1, pp. 119-124, Feb. 2004.

[44] Yang Zhou and Tao Jiang. A Novel Multi-points Square Mapping Combined with PTS for PAPR Reduction of OFDM Signals without Side Information; Appear to IEEE Transactions on Broadcasting.

[45] Z. Latinovic and Y. B. Ness. SFBC MIMO-OFDM peak-to-average power ratio reduction by polyphase interleaving and inversion; IEEE Communications Letters, vol. 10, no. 4, pp. 266-268, Apr. 2006.

[46] S. H. Han and J. H. Lee. Modified selected mapping technique for PAPR reduction of coded OFDM signal; IEEE Transactions on Broadcasting,vol. 50, no. 3, pp. 335-341, Sept. 2004.

[47] K. D. Choe, S. C. Kim and S. K. Park. Pre-scrambling method for PAPR reduction in OFDM communication systems; IEEE Transactions on Consumer Electronics, vol. 50, no. 4, pp. 1044-1048, Nov. 2004.

[48] S. Litsyn and G. Wunder. Generalized bounds on the crest-Factor distribution of OFDM signals with applications to code design; IEEE Transactions on Information Theory, vol. 52, no. 3, pp. 992-1006, Mar. 2006

[49] X. Li and L. J. Cimini. Effects of Clipping and Filtering on the Performance of OFDM; IEEE Communications Letters, vol. 2, no. 5, pp. 131-133, May. 1998.

[50] G. Santella and F. Mazzenga. A hybrid analytical-simulation procedure for performance evaluation in M-QAM-OFDM schemes in presence of nonlinear distortions; IEEE Transactions on Vehicular Technology, vol. 47, no. 1, pp. 142-151, Feb. 1998.

[51] A. Bahai and B. Saltzberg. Multi-Carrier Digital Communications: Theory and Applications of OFDM; Norwell, MA/New York: Kluwer/Plenum, 1999.

[52] M. Friese. On the achievable information rate with peak-power limited OFDM; IEEE Transactions on Information Theory, vol. 46, no. 11, pp. 2579-2587, Nov. 2000.

[53] N. Dinur and D. Wulich. Peak-to-Average Power Ratio in High-Order OFDM; IEEE Transactions on Communications, vol. 49, no. 6, pp. 10631072, Jun. 2001.

[54] S. Q. Wei, D. L. Goeckel and P. E. Kelly. A modem extreme value theory approach to calculating the distribution of the peak-to-average power ratio in OFDM systems; Prof. of IEEE International Conferences on Communications (ICC'02), vol. 3, pp. 1686-1690, Apr. 2002.

[55] S. M. Berman. Asymptotic independence of numbers of high and low level crossings of stationary Gaussian processes; The Annals of Mathematical Statistics, vol. 42, no. 3, pp. 927-945, 1971.

[56] M. R. Leadbetter and H. Rootzen. Extremal theory for stochastic processes; Annals of Probability, vol. 16, pp. 431-78, Apr. 1988.

[57] S. Q. Wei, D. L. Goeckel and P. A. Kelly. The complex envelope of bandlimited OFDM signals is asymptotically Gaussian: proof and application; http://www.ece.lsu.edu/swei/, 2006. 
[58] H. Yu, M. Chen and G. Wei. Distribution of PAR in DMT systems; Electronics Letters, vol. 39, no. 10, pp. 799-801, May. 2003.

[59] Gerhard Wunder and Holger Boche. Upper Bounds on the Statistical Distribution of the Crest-Factor in OFDM Transmission; IEEE Transactions on Information Theory, vol. 49, no. 2, pp. 488-494, Feb. 2003.

[60] G. Song and Y. Li. Adaptive subcarrier and power allocation in OFDM based on maximizing utility; Proc. of IEEE Vehicular Technology Conference (VTC'03), vol. 2, pp. 905-909, Apr. 2003.

[61] Tao Jiang, Mohsen Guizani, Hsiao-Hwa Chen, Weidong Xiang and Yiyan Wu. Derivation of PAPR Distribution for OFDM Wireless Systems Based on Extreme Value Theory; IEEE Transactions on Wireless Communications, vol. 7, no. 4, pp. 1298-1305, Apr. 2008.

[62] D. Kivanc, G. Q. Li and H. Liu. Computationally Efficient Bandwidth Allocation and Power Control for OFDMA; IEEE Transactions on Wireless Communications, vol. 2, no. 6, pp. 1150-1158, Nov. 2003.

[63] J. Jang and K. B. Lee. Transmit Power Adaptation for Multiuser OFDM systems; IEEE Journal on Selected Areas in Communications, vol. 21, no. 2, pp. 171-178, Feb. 2003.

[64] J. Campello de Souza. Discrete bit loading for multicarrier modulation systems; PhD thesis, University of Stanford, 1999.

[65] J. M. Cioffi. A multicarrier primer; ANSI TIE1.4 Committee Contribution, pp. 1-17, Nov. 1991. 